\title{
Point-ahead analysis and pre-pointing link stability study of intersatellite laser communication
}

\author{
FU-RUI ZHANG ${ }^{1,2^{*}}$, JUN-FENG HAN ${ }^{1}$, PING RUAN ${ }^{1}$ \\ ${ }^{1} \mathrm{Xi}$ 'an Institute of Optics and Precision Mechanics, Chinese Academy of Sciences, \\ Xi' an 710119, China \\ ${ }^{2}$ University of Chinese Academy of Sciences, Beijing 100049, China \\ *Corresponding author: jiffring@126.com
}

\begin{abstract}
The static bias error angle obviously affects pre-pointing links' stability in the presence of vibration in intersatellite laser communication. The 2 nd order point-ahead angle is a source of misalignment which was ignored in most solutions, and this is the concern of our paper. In this study, we present a further analytical investigation into the point-ahead angle in complex satellite maneuvering environment. Static bias error angle induced by the 2 nd order point-ahead angle has been studied under different intersatellite links. The probability density function of the pre-pointing links' outage has been derived in the presence of pointing jitter taking consideration of the static bias angle, and the link budget has also been analyzed. Simulation model of link stability has been established to verify the numerical results by the Monte Carlo method in Matlab-Simulink environment. The results have shown that the 2 nd order point-ahead angle has a significant detrimental impact on link stability in long distance links. It is a neglectable factor. This work is dedicated to intersatellite laser communication system design.
\end{abstract}

Keywords: intersatellite laser communication, point-ahead, link stability, simulation.

\section{Introduction}

With the advantages of high data rates, a small size and low power are in demand. Intersatellite laser link (ISLL) technology has been widely studied [1-ㅗ]. Several ISLLs have been first established such as ARTEMIS-SPOT4, OICETS-ARTEMIS, and NFIRE-TerraSAR-X [느므. In recent years, with the increase in detection distance in deep space missions, pointing link stability becomes a crucial issue. For instance, when communicating, it needs a point-ahead angle to compensate for the relative motion between the two satellites. On the other hand, the beam width of a pre-pointing link is only several microradians. With such a narrow beam width and over a distance of tens of thousands kilometers between the transmitter and the receiver, even a small sway 
of satellites may cause a significant pointing error. The pointing error angle consists of two parts, which are a dynamic error (induced by satellite jitter) and a static error (induced by misalignment). The distribution of random pointing errors has been introduced [7]. The precision of the point-ahead angle which is a source of misalignment decides whether the communication link can be established successfully or not in the pre-pointing stage (namely communication stage).

It is believed that jitter will reduce the pointing accuracy of a point-ahead link. Many significant researches on satellite jitter have been conducted [ $\underline{8}-\underline{10}]$. But so far, a detailed research of the point-ahead static bias error and its influence on pre-pointing link stability have not been conducted theoretically. There are kinds of errors which give rise to static bias errors, such as optical axis misalignment error, deformation error caused by temperature [11], etc. But there are few publications about calculating errors of the point -ahead in ISLL. Previously, researchers have done a great deal of studies on point-ahead links. However, in many of their calculation models the satellites have been treated as uniformly moving objects [12-14]. The 2 nd order point-ahead angle engendered by satellite acceleration was neglected. Under such conditions, the inaccuracy of the point-ahead angle will induce misalignment of the optic axis which may contribute to static bias pointing error. Therefore, it is necessary to make further studies of the stability of pre-pointing links in the presence of satellite jitter, taking into consideration the 2 nd order point-ahead static error angle.

Motivated by a reliable intersatellite link design, some meaningful researches have been presented in this paper. First of all, the calculation model of the point-ahead angle of ISLL has been established in Section 2. Then, the relationship between the point -ahead angle and the satellite maneuvering characteristics has been analyzed. Meanwhile, various regulations of the 2 nd order point-ahead angle in different orbit links have been given in Section 3. Furthermore, the probability density function (PDF) of the pre-pointing link outage has been derived in the presence of pointing jitter, taking into consideration the 2 nd order point-ahead angle and the link budget which is indispensable (Section 4). Finally, some numerical simulations have been deduced by the Monte Carlo method in Matlab-Simulink environment in Section 5. This work can improve the optical design of intersatellite laser communication.

\section{Calculation model of the intersatellite point-ahead angle}

Obviously, the point-ahead angle has a strong correlation with satellites' motion. Different links have distinctive characteristics. Usually, the point-ahead angle of GEO-GEO (geosynchronous Earth orbit) links and GEO-ground links is a fixed value. On the contrary, because LEO (low Earth orbit) satellites' maneuverability is complicated and there is a need that the two satellites see each other during communicating, the point-ahead angle of links which use LEO satellites is more complicated. Therefore, a LEO-LEO link has been chosen as the study model in this section. 


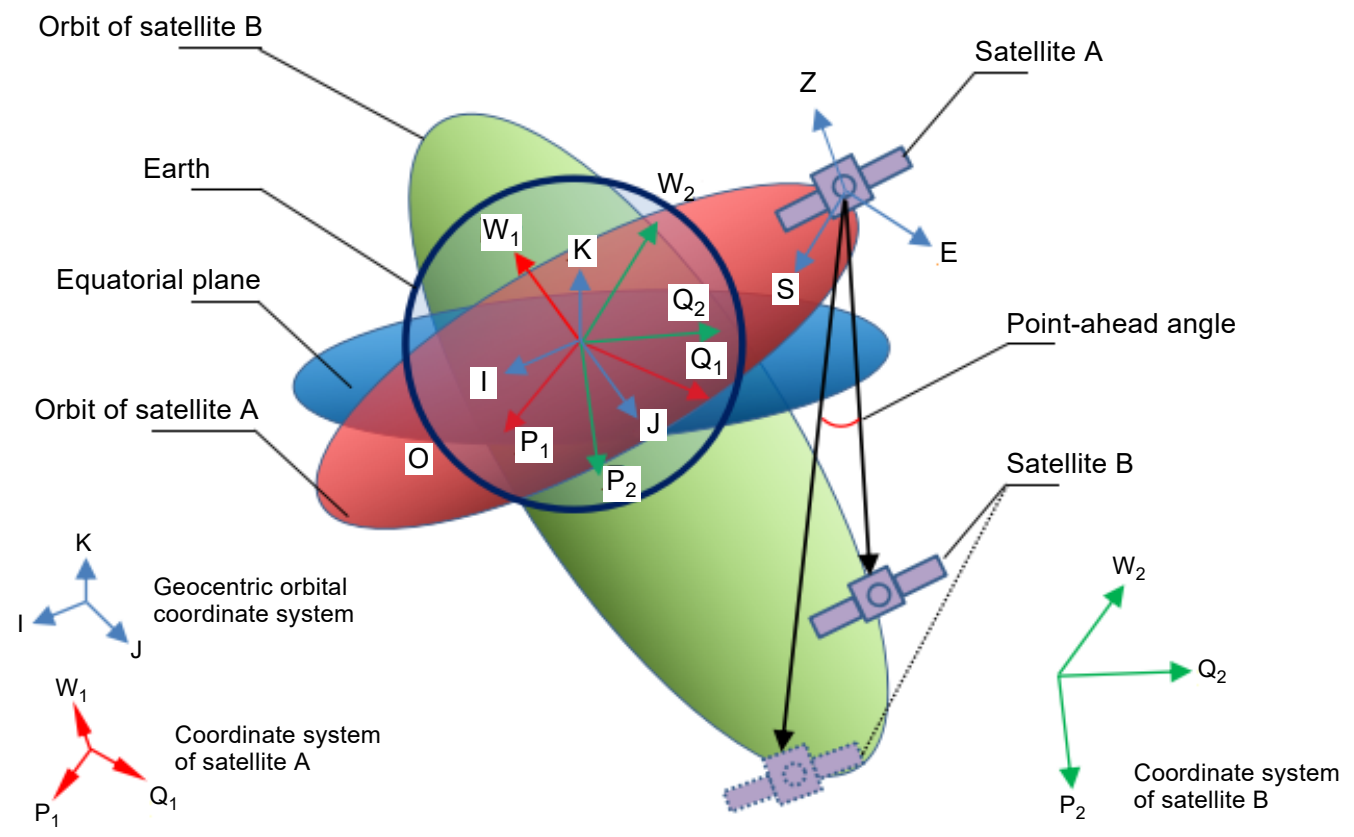

Fig. 1. The orbit model of intersatellite laser communication.

In order to achieve a point-ahead angle, the primary task is to calculate the motion information (position, speed and acceleration) of the two satellites. Here, we used the Kepler orbit elements method. The relative motion information in the equatorial coordinate system of the Earth can be obtained by numerical calculation. Then, the relative motion information must be converted to the master satellite's coordinate system, because the final executive mechanism of the point-ahead angle is the APT (acquisition pointing and tracking) system. Therefore, what must be done is to convert the motion information to the coordinate system of the terminal. Subsequently, we could derive the point-ahead angle calculation model. The schematic diagram of the intersatellite laser communication link coordinate system was depicted in Fig. 1. Thus, the link of the master satellite A points to the slave satellite B as a research model which involves three coordinate systems: geocentric orbit coordinate system O-PQW, geocentric equatorial coordinate system O-IJK and satellite elevation/azimuth coordinate system O-SEZ.

Pre-pointing link is a time continuous process. The point-ahead angle at any moment could be derived from the ephemeral data. Compared to the interpolation method, the Kepler orbit elements method (KOEM) had a higher accuracy. The KOEM requires six orbital elements, which are orbit inclination angle $i$, perigee angle $\omega$, orbital eccentricity $e$, ascending ascension $\Omega$, semi-major axis $a$, and true anomaly $f$. The true anomaly angle is acquired by solving the Kepler equation. Then, the position, velocity and acceleration of the two satellites can be expressed as 


$$
\left\{\begin{array}{l}
\mathbf{r}_{\mathrm{PQW}}=\left[\begin{array}{lll}
x & y & z
\end{array}\right]^{\mathrm{T}} \\
\dot{\mathbf{r}}_{\mathrm{PQW}}=\left[\begin{array}{lll}
\dot{x} & \dot{y} & \dot{z}
\end{array}\right]^{\mathrm{T}} \\
\ddot{\mathbf{r}}_{\mathrm{PQW}}=\left[\begin{array}{lll}
\ddot{x} & \ddot{y} & \ddot{z}
\end{array}\right]^{\mathrm{T}}
\end{array}\right.
$$

where $\mathbf{r}_{\mathrm{PQW}}, \dot{\mathbf{r}}_{\mathrm{PQW}}$, and $\ddot{\mathbf{r}}_{\mathrm{PQW}}$ are position, velocity and acceleration of the two satellites in a near focus coordinate system. The specific expressions about $\mathbf{r}_{\mathrm{PQW}}, \dot{\mathbf{r}}_{\mathrm{PQW}}$, and $\ddot{\mathbf{r}}_{\mathrm{PQW}}$ can be found in [15]. Because the satellite motion information obtained by $\mathrm{KOEM}$ is under the non-inertia coordinate system O-PQW which is a dynamic coordinate system, vector operations cannot be performed. So it is necessary to transform $\mathbf{r}_{\mathrm{PQW}}, \dot{\mathbf{r}}_{\mathrm{PQW}}$, and $\ddot{\mathbf{r}}_{\mathrm{PQW}}$ into the geocentric equatorial coordinate system O-IJK:

$$
\left\{\begin{array}{l}
\mathbf{r}_{\mathrm{IJK}}=\left[\begin{array}{lll}
x^{\prime} & y^{\prime} & z^{\prime}
\end{array}\right]^{\mathrm{T}}=\tilde{R} \mathbf{r}_{\mathrm{PQW}} \\
\dot{\mathbf{r}}_{\mathrm{IJK}}=\left[\begin{array}{lll}
\dot{x}^{\prime} & \dot{y}^{\prime} & \dot{z}^{\prime}
\end{array}\right]^{\mathrm{T}}=\tilde{R} \dot{\mathbf{r}}_{\mathrm{PQW}} \\
\ddot{\mathbf{r}}_{\mathrm{IJK}}=\left[\begin{array}{lll}
\ddot{x}^{\prime} & \ddot{y}^{\prime} & \ddot{z}^{\prime}
\end{array}\right]^{\mathrm{T}}=\tilde{R} \ddot{\mathbf{r}}_{\mathrm{PQW}}
\end{array}\right.
$$

where $\mathbf{r}_{\mathrm{IJK}}, \dot{\mathbf{r}}_{\mathrm{IJK}}$, and $\ddot{\mathbf{r}}_{\mathrm{IJK}}$ are position, velocity and acceleration of the two satellites in the geocentric orbital coordinate system and $\tilde{R}$ is the transformation matrix [16]. Then, the relative position, velocity and acceleration between the master satellite and the slave satellite can be obtained by vector operations under the geocentric equatorial inertial coordinate system:

$$
\left\{\begin{array}{l}
\mathbf{r}_{\mathrm{AB}}=\mathbf{r}_{\mathrm{IJK}}^{\mathrm{A}}-\mathbf{r}_{\mathrm{IJK}}^{\mathrm{B}} \\
\dot{\mathbf{r}}_{\mathrm{AB}}=\dot{\mathbf{r}}_{\mathrm{IJK}}^{\mathrm{A}}-\dot{\mathbf{r}}_{\mathrm{IJK}}^{\mathrm{B}} \\
\ddot{\mathbf{r}}_{\mathrm{AB}}=\ddot{\mathbf{r}}_{\mathrm{IJK}}^{\mathrm{A}}-\ddot{\mathbf{r}}_{\mathrm{IJK}}^{\mathrm{B}}
\end{array}\right.
$$

In Eq. (3), $\mathbf{r}_{\mathrm{AB}}, \dot{\mathbf{r}}_{\mathrm{AB}}$, and $\ddot{\mathbf{r}}_{\mathrm{AB}}$ are relative position, relative velocity and relative acceleration of the satellite $B$ with respect to the satellite A. At last, the point-ahead angle is represented in the coordinate system O-SEZ on the satellite payload. So, what still must be done is to convert $\mathbf{r}_{\mathrm{AB}}, \dot{\mathbf{r}}_{\mathrm{AB}}$, and $\ddot{\mathbf{r}}_{\mathrm{AB}}$ into O-SEZ coordinate system:

$$
\left\{\begin{array}{l}
\left\langle\oplus \mathbf{r}_{\mathrm{AB}}\right\rangle=\left[\begin{array}{lll}
X_{\mathrm{AB}^{*}} & Y_{\mathrm{AB}^{*}} & Z_{\mathrm{AB}}{ }^{\mathrm{T}}
\end{array}\right]^{\mathrm{T}}=\tilde{U} \mathbf{r}_{\mathrm{AB}} \\
\left\langle\oplus \dot{\mathbf{r}}_{\mathrm{AB}}\right\rangle=\left[\begin{array}{lll}
\dot{X}_{\mathrm{AB}} & \dot{Y}_{\mathrm{AB}} & \dot{Z}_{\mathrm{AB}}
\end{array}\right]^{\mathrm{T}}=\tilde{U} \dot{\mathbf{r}}_{\mathrm{AB}} \\
\left\langle\oplus \ddot{\mathbf{r}}_{\mathrm{AB}}\right\rangle=\left[\begin{array}{lll}
\ddot{X}_{\mathrm{AB}} & \ddot{Y}_{\mathrm{AB}} & \ddot{Z}_{\mathrm{AB}}
\end{array}\right]^{\mathrm{T}}=\tilde{U} \ddot{\mathbf{r}}_{\mathrm{AB}}
\end{array}\right.
$$

Here $\oplus \mathbf{r}_{\mathrm{AB}}, \oplus \dot{\mathbf{r}}_{\mathrm{AB}}$, and $\oplus \ddot{\mathbf{r}}_{\mathrm{AB}}$ are relative position, relative velocity and relative acceleration of the satellite $\mathrm{B}$ with respect to the satellite A in the terminal's coordinate 
system, and $\tilde{U}$ is the transformation matrix. Assuming that the satellite and the Earth constitute a two-body model in which the perturbation force was ignored, the relative displacement components on $X, Y$, and $Z$ axis can be expressed as:

$$
\left\{\begin{array}{l}
\left\langle X_{\mathrm{AB}^{*}}\right\rangle=\dot{X}_{\mathrm{AB}^{*}} \Delta t+\frac{1}{2} \ddot{X}_{\mathrm{AB}} \Delta t^{2} \\
\left\langle Y_{\mathrm{AB}}\right\rangle=\dot{Y}_{\mathrm{AB}^{*}} \Delta t+\frac{1}{2} \ddot{Y}_{\mathrm{AB}} * \Delta t^{2} \\
\left\langle Z_{\mathrm{AB}}\right\rangle=\dot{Z}_{\mathrm{AB}} * \Delta t+\frac{1}{2} \ddot{Z}_{\mathrm{AB} *} \Delta t^{2}
\end{array}\right.
$$

where $\Delta t$ is the relaxation time. By substituting Eqs. (1)-(4) into Eqs. (5) we can obtain the expressions for azimuth and elevation angles, azimuth and elevation of the 1st order point-ahead angle and azimuth and elevation of the 2 nd order point-ahead angle:

$$
\begin{aligned}
& P_{\theta_{\mathrm{az}}}= \begin{cases}\arctan \left(\frac{Y_{\mathrm{AB}^{*}}}{X_{\mathrm{AB}^{*}}}\right)-\frac{\pi}{2}, & \frac{Y_{\mathrm{AB}^{*}}}{X_{\mathrm{AB}}} \geq 0 \\
\arctan \left(\frac{Y_{\mathrm{AB}^{*}}}{X_{\mathrm{AB}^{*}}}\right)+\frac{3 \pi}{2}, & \frac{Y_{\mathrm{AB}^{*}}}{X_{\mathrm{AB}^{*}}}<0\end{cases} \\
& P_{\theta_{\mathrm{el}}}=-\arctan \left(\frac{Z_{\mathrm{AB}^{*}}}{\sqrt{\dot{X}_{\mathrm{AB}}^{2}+\dot{Y}_{\mathrm{AB}}^{2}}}\right)
\end{aligned}
$$

Azimuth and elevation of the 1st order point-ahead angle is:

$$
\begin{aligned}
& \dot{P}_{\theta_{\mathrm{az}}}= \begin{cases}\arctan \left(\frac{\dot{Y}_{\mathrm{AB}^{*}}}{\dot{X}_{\mathrm{AB}^{*}}}\right)-\frac{\pi}{2}, & \frac{\dot{Y}_{\mathrm{AB}^{*}}}{\dot{X}_{\mathrm{AB}}} \geq 0 \\
\arctan \left(\frac{\dot{Y}_{\mathrm{AB}^{*}}}{\dot{X}_{\mathrm{AB}^{*}}}\right)+\frac{3 \pi}{2}, & \frac{\dot{Y}_{\mathrm{AB}^{*}}}{\dot{X}_{\mathrm{AB}^{*}}}<0\end{cases} \\
& \dot{P}_{\theta_{\mathrm{el}}}=-\arctan \left(\frac{\dot{Z}_{\mathrm{AB}^{*}}}{\sqrt{\dot{X}_{\mathrm{AB}}^{2}+\dot{Y}_{\mathrm{AB}}^{2}}}\right)
\end{aligned}
$$

and azimuth and elevation of the 2 nd order point-ahead angle is: 


$$
\begin{aligned}
& \ddot{P}_{\theta_{\mathrm{az}}}= \begin{cases}\arctan \left(\frac{\ddot{Y}_{\mathrm{AB}^{*}}}{\ddot{X}_{\mathrm{AB}^{*}}}\right)-\frac{\pi}{2}, & \frac{\ddot{Y}_{\mathrm{AB}^{*}}}{\ddot{X}_{\mathrm{AB}^{*}}} \geq 0 \\
\arctan \left(\frac{\ddot{Y}_{\mathrm{AB}^{*}}}{\ddot{X}_{\mathrm{AB}}}\right)+\frac{3 \pi}{2}, & \frac{\ddot{Y}_{\mathrm{AB}^{*}}}{\ddot{X}_{\mathrm{AB}}}<0\end{cases} \\
& \ddot{P}_{\theta_{\mathrm{el}}}=-\arctan \left(\frac{\ddot{Z}_{\mathrm{AB}^{*}}}{\sqrt{\ddot{X}_{\mathrm{AB}^{*}}^{2}+\ddot{Y}_{\mathrm{AB}}^{2}}}\right)
\end{aligned}
$$

\section{Analysis}

\subsection{Analysis of point-ahead and satellites maneuverability}

In this section, a further comprehension of the relation between the point-ahead angle and the satellite motion features was studied under the tow-body model without considering satellite perturbation for the reason that the link duration time is very short. Furthermore, we analyzed the 2 nd order static error angle $\varphi$ which changes regulation in different orbit links. According to the calculation model established in Section 2, we have chosen two LEO (low Earth orbits) satellites to prepare the link model for the analysis. The orbit elements of the two satellites are summarized in Table 1.

T a b l e 1. Simulation model-orbit elements.

\begin{tabular}{lllllll}
\hline & $i$ & $e$ & $a$ & $\Omega$ & $\omega$ & $f$ \\
\hline Satellite A & $67^{\circ}$ & 0.0014435 & $622 \mathrm{~km}$ & $334.5^{\circ}$ & $45^{\circ}$ & $240^{\circ}$ \\
Satellite B & $34^{\circ}$ & 0.0002053 & $508 \mathrm{~km}$ & $253^{\circ}$ & $91.2^{\circ}$ & $300^{\circ}$ \\
\hline
\end{tabular}

The initial time of the two satellites was 9:27:30 on September 6, 2018. The intervisible time of two satellites lasted 1500 seconds. The numerical analysis of slant, optical relaxation time, azimuth and elevation angle, azimuth and elevation angle speed, azimuth and elevation of the 1st order point-ahead angle, azimuth and elevation angle acceleration and azimuth and elevation of the 2 nd order point-ahead angle were evaluated, respectively. The results are shown in Fig. 2.

As the results in Fig. 2a show, the slant distance reached a minimum value of $916 \mathrm{~km}$ at a route shortcut point and the light relaxation time reached a minimum value of $0.003 \mathrm{~s}$ at the same time. Simultaneously, in Fig. 2b, the azimuth and elevation angle reached the same value. In Fig. 2c , the maximum value of the azimuth and elevation angle speed are -0.35 and $0.33 \mathrm{deg} / \mathrm{s}$, respectively. The 1 st order point-ahead angle was inversely proportional to the slant. This regulation is consistent with Shimizu's conclusion [12]. From Fig. 2d, we can find that the azimuth and elevation of 2 nd order point-ahead angle both reached zero at a route shortcut point. Meanwhile, the 2 nd order point-ahead 

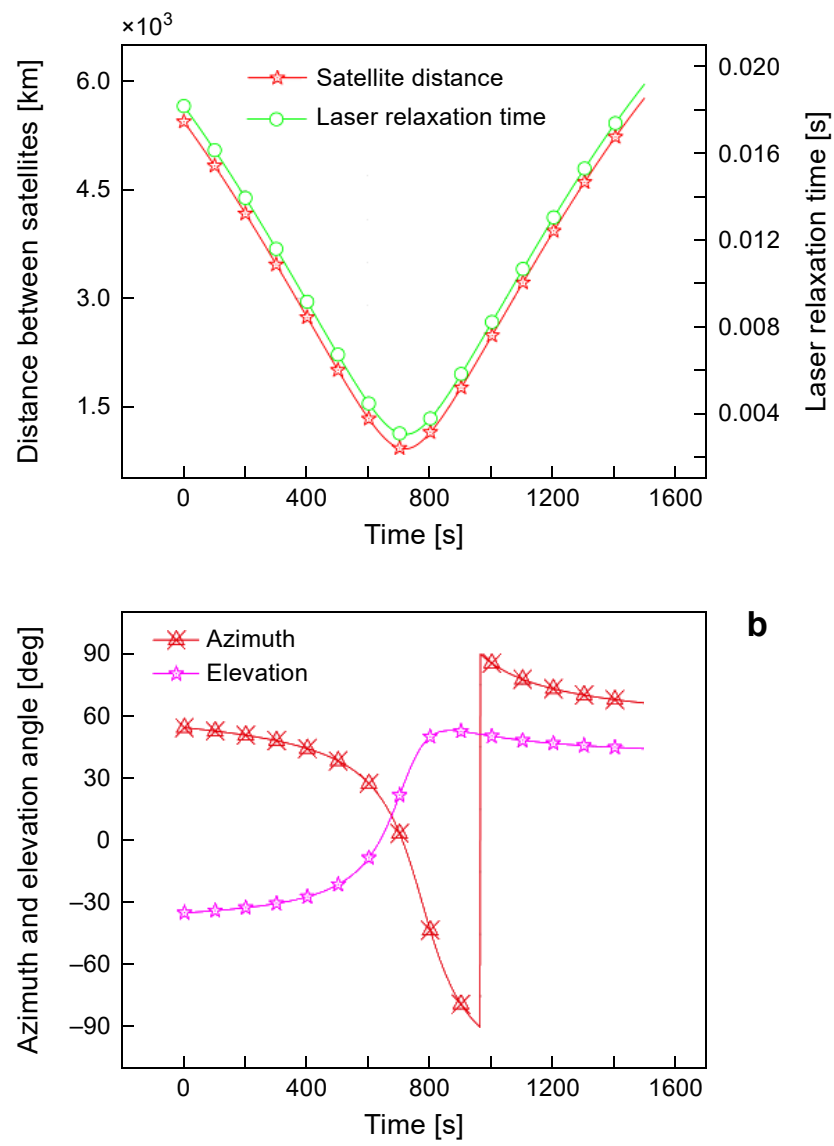

b

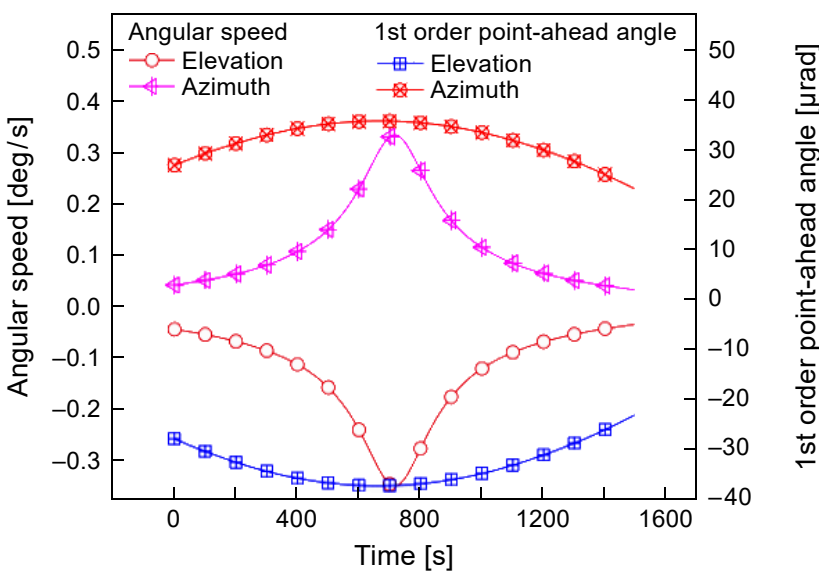

C

Fig. 2. Satellites maneuverability and point-ahead. Satellites distance and optical relaxation time (a), angle of azimuth and elevation (b), azimuth, elevation angle speed and the 1st order point-ahead angle (c), and azimuth, elevation angle acceleration and the 2 nd order point-ahead angle (d). 

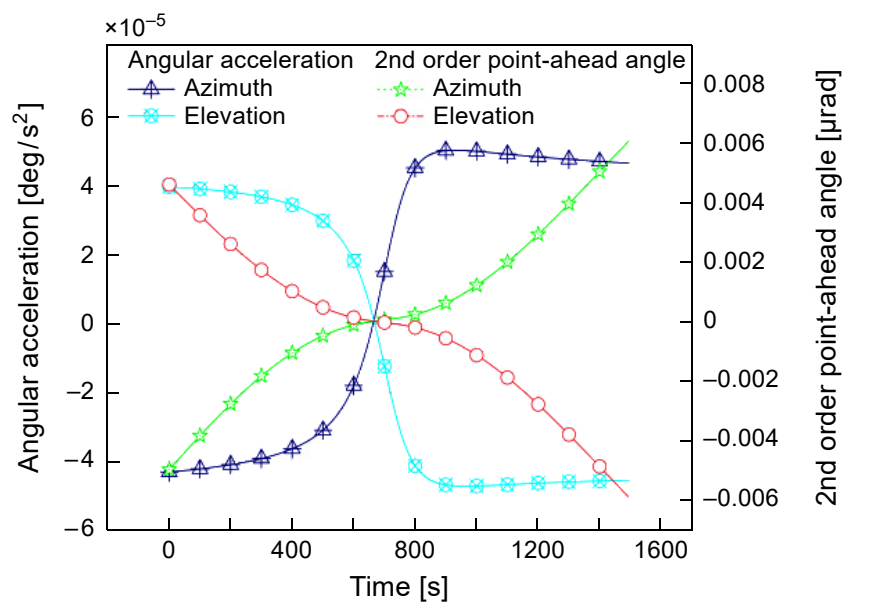

Fig. 2. Continued.

is proportional to intersatellite slant and it differs from that of the 1 st order point-ahead angle. The maximum point-ahead angle in our model is about $40 \mu \mathrm{rad}$, which is less than the estimated value $50 \mu \mathrm{rad}$ in [1ㅜㅇ.

From the above analysis, we can draw a conclusion that the 1 st order point-ahead angle will not incur critical issues in some ISLLs. The 2 nd order point-ahead angle is proportional to the slant distance. Since the relative motion in ISLL which consists of different types of orbits of satellites are polytropical, the slant distance has a wide span. Therefore, the 2 nd order point-ahead angle will induce an obvious static error angle. Consequently, it is necessary to analyze the 2 nd order point-ahead angle in different types of ISLLs.

\subsection{Second order point-ahead analysis}

Among the six orbital parameters, only the change of $a, i, \omega$ and $\Omega$ can give rise to the variation of slant. We changed the orbital parameters of $a, i, \omega$ and $\Omega$ of the slave satellite B. Each variable has three sets of values and each group of experiments lasted $1600 \mathrm{~s}$. The azimuth and elevation of the 2 nd order point-ahead angle in different ISLLs can be achieved in Fig. 3 .

In Fig. 3a, orbit height increased 74.2 times from 512 to $37979 \mathrm{~km}$. The maximum value of azimuth and elevation of the 2 nd order point-ahead angle increased by 16.4 and 65 times, respectively. The maximum of the 2 nd point-ahead angle is $0.6 \mu \mathrm{rad}$ under the link distance of $37979 \mathrm{~km}$. From Fig. 3b, we can find that the elevation of the 2 nd order point-ahead angle changed with inclination, obviously. Figure $3 \mathbf{c}$ shows that both the azimuth and elevation of the 2 nd order point-ahead angle have a weak correlation with RAAN (right ascension of the ascending node). In Fig. $3 \mathbf{d}$ we can find that azimuth of the 2 nd order point-ahead angle has a strong correlation with perigee. 

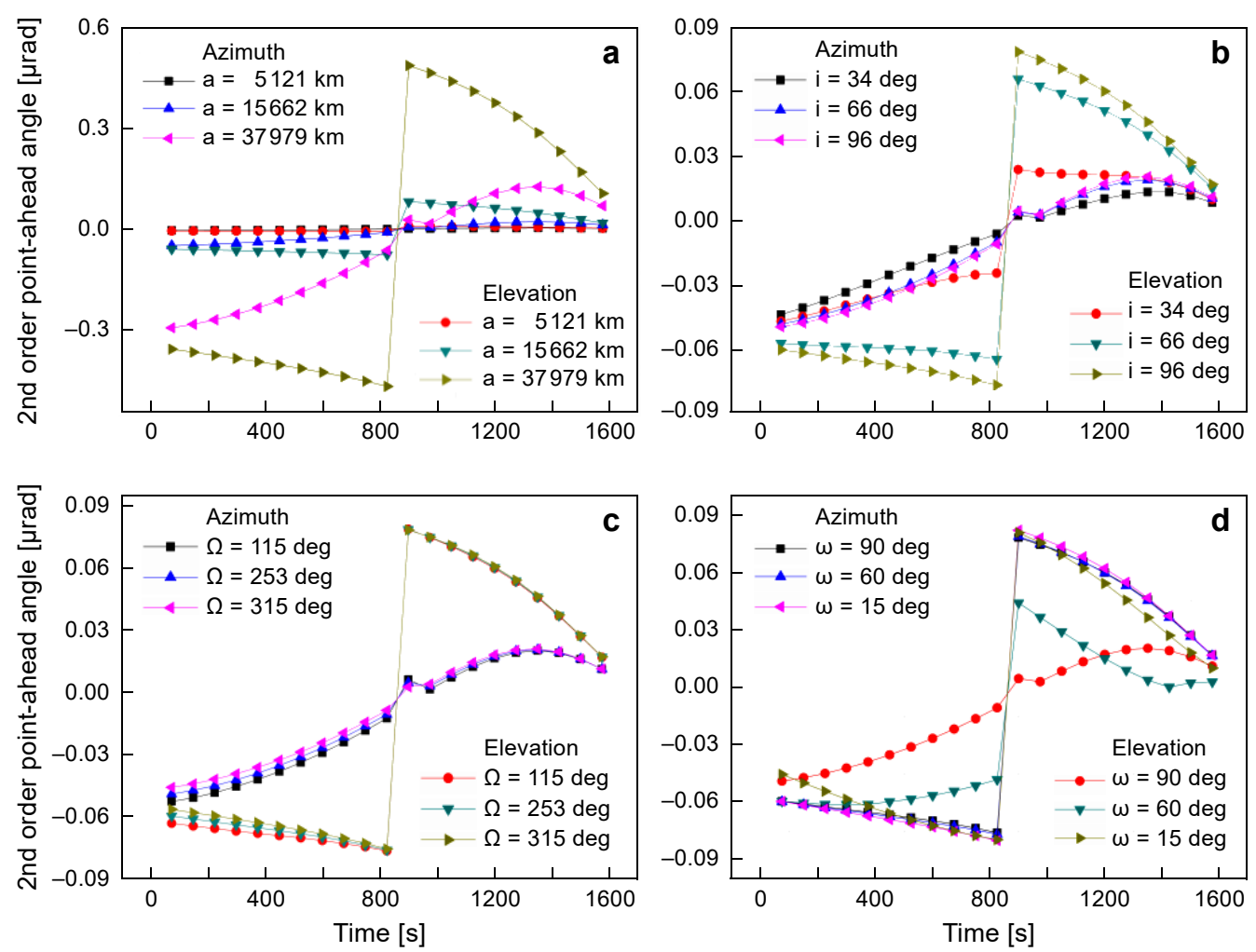

Fig. 3. Second order point-ahead angle vary characteristics in different orbit links: different orbit height (a), different orbit inclination (b), different RAAN (c), and different perigee accessory angle (d)

As discussed above, the satellite was considered as a uniform moving object in many solutions in which the 2 nd order point-ahead angles were ignored. Along with the increase in the link distance, the static bias error angle induced by the 2 nd order point-ahead angle will damage the pre-pointing link. Simultaneously, random jitter also exists on the satellite. Under such adverse conditions, using a narrow divergence beam (generally is 10-20 $\mu \mathrm{rad}$ ) in pre-pointing links may be arduous. On the other hand, so far a detailed analysis of link outage probability in the presence of the 2 nd order point-ahead angle (taking jitter into consideration) has not been conducted theoretically.

\section{Link stability analysis}

\subsection{Link outage probability model}

Figure $4 \mathbf{a}$ showed the pointing error angle principle. The pre-pointing model can be seen in Fig. $4 \mathbf{b}$. Satellite A is the master satellite, while satellite B is the slave satellite. Parameter $\theta$ represents the communication laser beam width. Assume that $\theta_{\mathrm{T}}$ repre- 


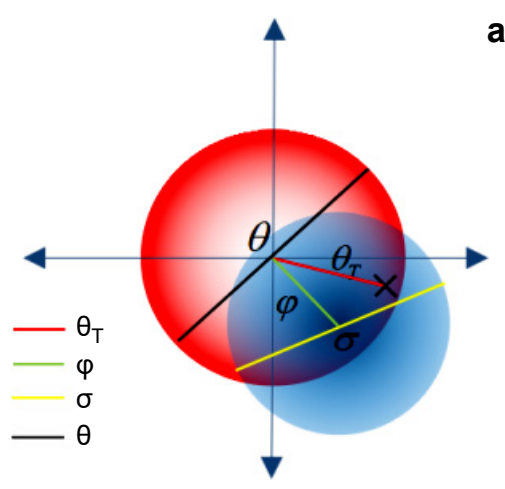

a

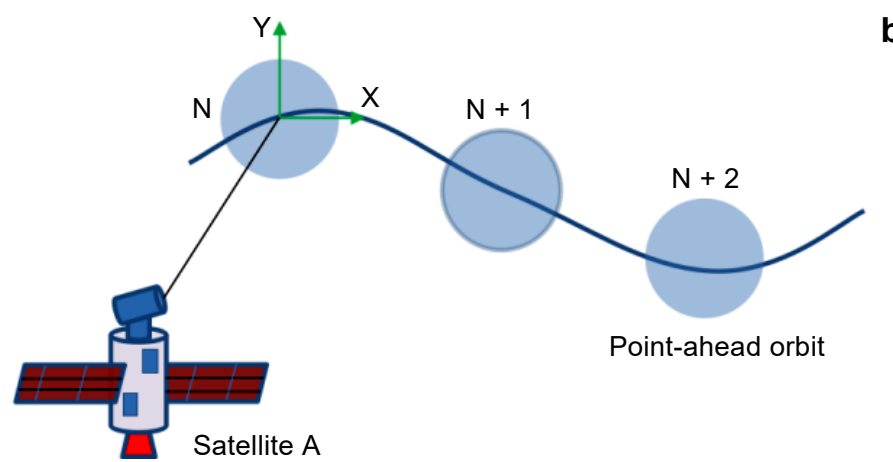

Fig. 4. Pointing error angle (a), and pre-pointing link (b) schematic diagrams.

sents the pointing error angle induced by the dynamic error angle $\sigma$ and static bias error angle $\varphi$. The pre-pointing link will break off if $\theta_{\mathrm{T}}$ exceeds the threshold error angle $\varepsilon^{*}$. The relationship between the threshold error angle $\varepsilon^{*}$ and the beam width $\theta_{\mathrm{p}}$ is given in [17].

Static bias error angle $\varphi$ represents the deviation between the mean optical axis position and the actual position. The dynamic error angle $\sigma$ represents the stability of the optical axis. The radial error of the pointing error $\theta_{\mathrm{T}}$ follows the Rician distribution with the assumption that the azimuth and elevation error are identical and uncorrelated, which is $[\underline{18}]$

$$
f\left(\theta_{\mathrm{T}}, \varphi\right)=\frac{\theta_{\mathrm{T}}}{\sigma^{2}} \exp \left\{-\frac{\theta_{\mathrm{T}}^{2}+\varphi^{2}}{2 \sigma^{2}}\right\} I_{0}\left(\frac{\theta_{\mathrm{T}}^{2} \varphi}{\sigma^{2}}\right)
$$

where $I_{0}(\cdot)$ represents the first kind of the zero order Bessel function. Then each of the pre-pointing link outage probability can be rewritten as

$$
P_{\text {outage }}\left(\sigma^{2}\left(\theta_{\mathrm{T}}^{2}\right)>\left(\varepsilon^{*}\right)^{2}\right)=\int_{\frac{\theta_{\mathrm{b}}}{4.88 q}}^{\infty} \frac{\theta_{\mathrm{T}}^{2}}{\sigma^{2}} \exp \left\{-\frac{\theta_{\mathrm{T}}^{2}+\varphi^{2}}{2 \sigma^{2}}\right\} I_{0}\left(\frac{\theta_{\mathrm{T}}^{2} \varphi}{\sigma^{2}}\right) \mathrm{d} \theta_{\mathrm{T}}
$$




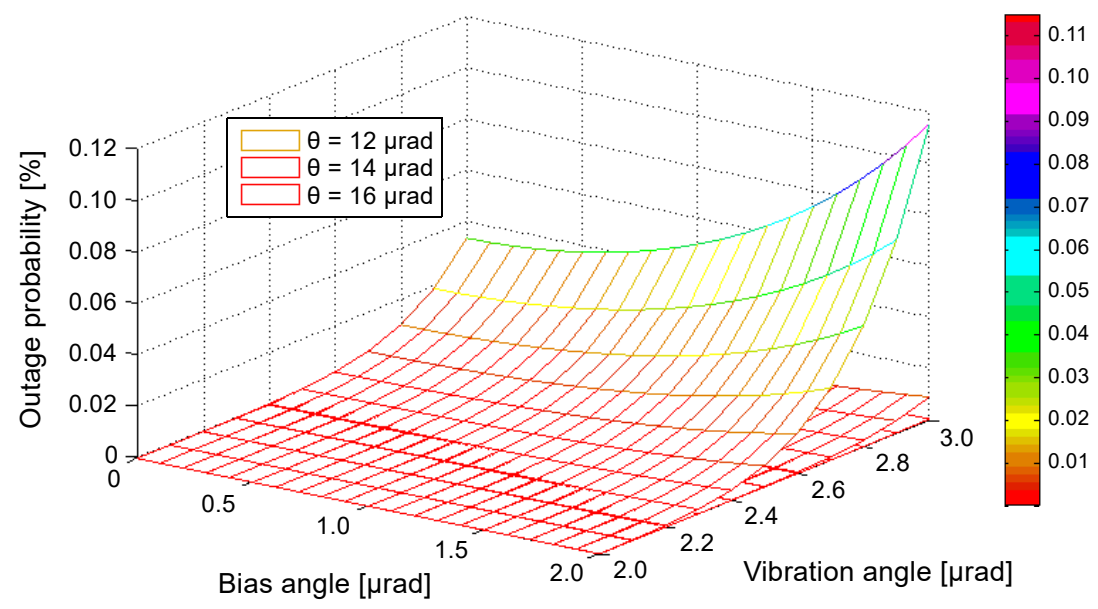

Fig. 5. Link outage probability increased exponentially with 2 nd order point-ahead angle.

Equation (10) is the CDF (cumulative distribution function) of link outage probability, namely $\theta_{\mathrm{T}}$ between $\varepsilon^{*}$ to $\infty$. In probability theories, the CDF of the Rician distribution is Marcum- $Q$-function which is

$$
Q_{\mathrm{M}}(a, b)=\exp \left(-\frac{a^{2}+b^{2}}{2}\right) \sum_{k=1-M}^{\infty}\left(\frac{a}{b}\right)^{k} I_{k}(a b)
$$

By substituting corresponding parameters into Eq. (11) and taken $(M-1)$, the equation can be rewritten as

$$
P_{\text {outage }}=Q_{1}\left(\frac{\varphi}{\sigma}, \frac{\theta_{\mathrm{T}}}{7.32 \sigma}\right)=\exp \left(-\frac{\theta_{\mathrm{T}}+53.58 \varphi^{2}}{107.16 \sigma^{2}}\right) I_{0}\left(\frac{\theta_{\mathrm{T}} \varphi}{7.32 \sigma^{2}}\right)
$$

The reason for the static bias error angle is manifold, such as alignment error, thermal deformation error and manufacturing error of optical components. We have taken the static bias error among 0-2 $\mu \mathrm{rad}$, and have taken satellite jitter among 2-3 $\mu \mathrm{rad}$. Beam width are 12,14 and $16 \mu \mathrm{rad}$, respectively, in three groups. Numerical simulation carried out in MATLAB and Fig. 5 showed the results. As one can see, under the same conditions, the effect of the jitter error angle on link outage probability was greater than that of the static bias error angle. On the other hand, with the increase in beam width, the probability of link outage decreased rapidly.

\subsection{Link budget}

Moreover, the pointing error has a significant effect on the intensity fluctuation. The link margin should be sufficient to withstand other power losses. We analyzed the link 
budget, and optical signal power at the receiver obtained by multiplying the transmitter power, telescope gain, and various losses are given as follows [19]:

$$
P_{\mathrm{R}}\left(\theta_{\mathrm{T}}\right)=P_{\mathrm{T}} \eta_{\mathrm{T}} G_{\mathrm{T}} L_{\mathrm{SB}} L_{\mathrm{T}}\left(\theta_{\mathrm{T}}\right) \eta_{\mathrm{R}} G_{\mathrm{R}}-\operatorname{SNR} P_{\mathrm{S}}
$$

where $P_{\mathrm{T}}$ is the mean transmitter optical power, $\eta_{\mathrm{T}}$ and $\eta_{\mathrm{R}}$ are optical system efficiency of the transmitter and receiver, respectively. Generally, the efficiency of the optical system is among $0.4-0.7$. We have chosen 0.5 in this paper. $G_{\mathrm{T}}$ is the transmitter telescope gain, and $G_{\mathrm{R}}$ is the receiver telescope gain. $L_{\mathrm{SB}}$ is the free space loss and $L_{\mathrm{T}}\left(\theta_{\mathrm{T}}\right)$ is the transmitter pointing loss. $P_{\mathrm{s}}$ is the sensitivity of the InGaAs APD detector and the value is $-65 \mathrm{dBm}$. We take the signal-to-noise ratio (SNR) of the detector as 7. The detailed expression of $P_{\mathrm{T}}, G_{\mathrm{T}}, L_{\mathrm{SB}}, L_{\mathrm{T}}\left(\theta_{\mathrm{T}}\right)$, and $G_{\mathrm{R}}$ is given in $[20,21]$. And then Eq. (13) can be rewritten as

$$
P_{\mathrm{R}}\left(\theta_{\mathrm{T}}\right)=P_{\mathrm{T}} \eta_{\mathrm{T}}\left(\frac{\pi D_{\mathrm{T}}}{\lambda}\right)^{2}\left(\frac{\lambda}{2 \pi z}\right)^{2} \exp \left(-G_{\mathrm{T}} \theta_{\mathrm{T}}^{2}\right) \eta_{\mathrm{R}}\left(\frac{\pi D_{\mathrm{R}}}{\lambda}\right)^{2}-\mathrm{SNR} P_{\mathrm{s}}
$$

The center wavelength is $1550 \mathrm{~nm}$, the link distance is $45000 \mathrm{~km}$ and the mean optical power of the transmitter is $10 \mathrm{~W}$. Assuming that the variance of the jitter error angle is $5 \mu \mathrm{rad}$ and the static bias error is $3 \mu \mathrm{rad}$, according to Eq. (7), the pointing error $\theta_{\mathrm{T}}$ is most likely $5 \mu \mathrm{rad}$. We substitute relevant parameters into Eqs. (12), and then we can obtain the link budget as given in Table 2 . As one can see, the link margin was only $0.62 \mathrm{~dB}$, which is insufficient for other losses. The $G_{\mathrm{T}}, G_{\mathrm{R}}$ and $L_{\mathrm{SB}}$ have fixed values, if the link distance and telescope diameter are determined. Thus, the feasible solution was to improve the pointing accuracy. In a word, the link bandwidth resources in a long ISLL was very short.

T a b l e 2. Pointing link power budget.

\begin{tabular}{lll}
\hline Symbol & Parameter name & Value \\
\hline$P_{\mathrm{T}}$ & Transmitter optical power & $10 \mathrm{~W}$ \\
$\eta_{\mathrm{T}}$ & Optics efficiency of the transmitter & $-3 \mathrm{~dB}$ \\
$G_{\mathrm{T}}$ & Transmitter telescope gain & $106 \mathrm{~dB}$ \\
$L_{\mathrm{SB}}$ & Free space propagation loss & $-291.2 \mathrm{~dB}$ \\
$\eta_{\mathrm{R}}$ & Receiver optical efficiency & -3 \\
$G_{\mathrm{R}}$ & Telescope gain of receiver & $106 \mathrm{~dB}$ \\
$z$ & Satellite distance & $45000 \mathrm{~km}$ \\
$L_{\mathrm{T}}(\theta)$ & Pointing error loss & -4.5 \\
$\lambda$ & Laser wavelength & $1.55 \mu \mathrm{m}$ \\
$D_{\mathrm{T}}, D_{\mathrm{R}}$ & Telescope diameter & $0.1 \mathrm{~m}$ \\
$\omega$ & Half width of divergence angle & $30 \mu \mathrm{rad}$ \\
& Link margin & $0.62 \mathrm{~dB}$ \\
$P_{\mathrm{R}}\left(\theta_{\mathrm{T}}\right)$ & Received power & $8.49 \times 10^{-9} \mathrm{~W}$ \\
\hline
\end{tabular}




\section{Simulink simulation}

Link maintenance time is an important indicator of pre-pointing link stability. The lower the outage probability of the communication link, the longer of the communication time. In order to verify the theory of link outage probability, the Monte Carlo method was used in Matlab-Simulink simulation model. Each point-ahead can be seen as an independent event, assuming that the detector's probe period is $\tau$, and the link outage probability of each pre-point is $p$, the outage probability distribution in time $N \tau$ obeys geometric distribution. The events number expectation of link outage probability is $E(X)=1 / p$. Accordingly, the time expectation is $E(X)=t / p[\underline{22}]$. The probability of link outage can be represented by the ration of the number of times that $\theta_{\mathrm{T}}$ exceed $\varepsilon^{*}$ to the total simulation number of times. The simulation model is depicted in Fig. 6 .

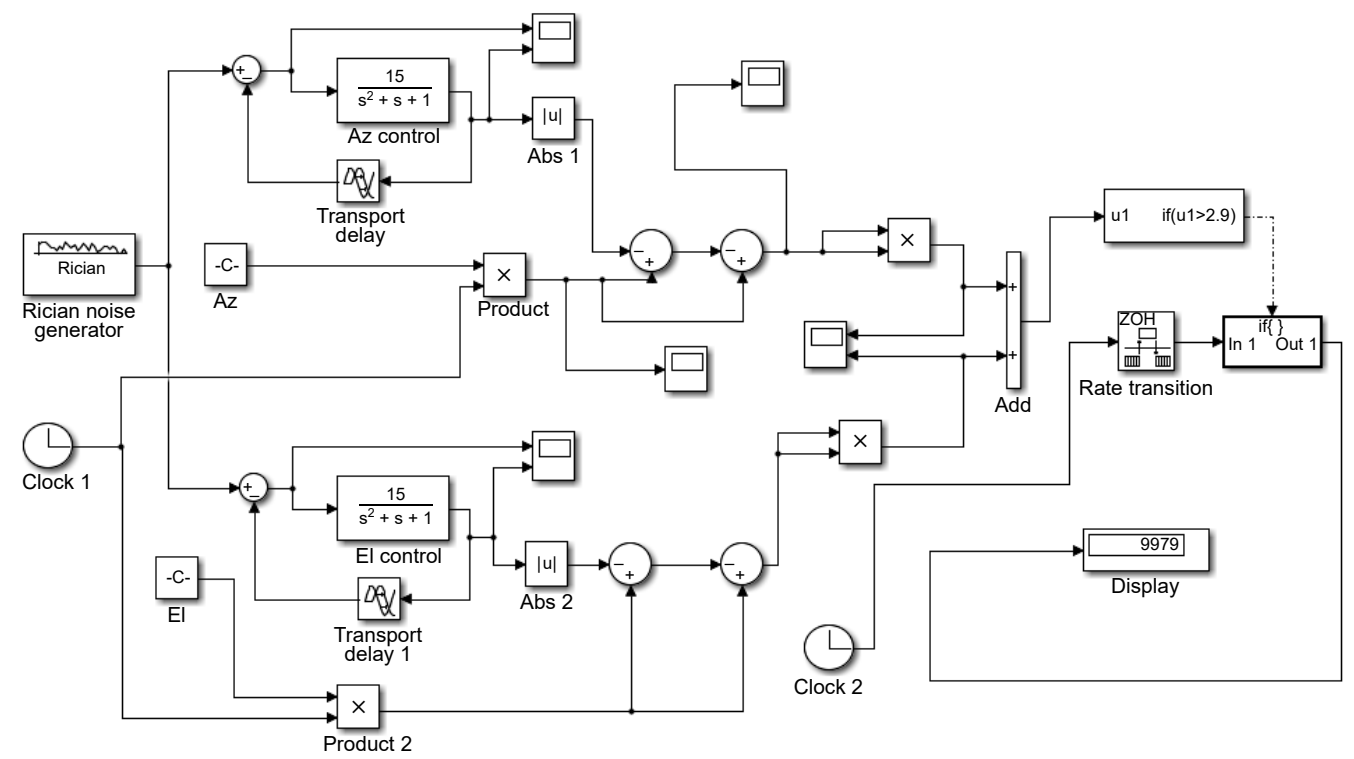

Fig. 6. Simulation model scheme.

In the pre-pointing link, a fast steering mirror (FSM) is used to control the beam pointing direction. The preferred control mode for the FSM is a closed-loop control method, and the transfer function model used in our system can be found in [23]. More than $50 \%$ of coarse tracking residual can be corrected by the PID controller. We can select different beam width, vibration error angle, static bias error angle and satellite motion velocity in this model. For convenience, here we take $\tau=1$ and the results will be nominal values. The results are shown in Fig. 7.

Simulation results of the link retention time versus the static bias error $\varphi$ for various jitter and beam width of $\sigma=3$ and $4 \mu \mathrm{rad}$ and $\theta=17,19,21$, and $25 \mu \mathrm{rad}$ are shown in Fig. 7. If the pointing error angle $\theta_{\mathrm{T}}$ is greater than $\theta / 6.9$, the link broke off. The results 

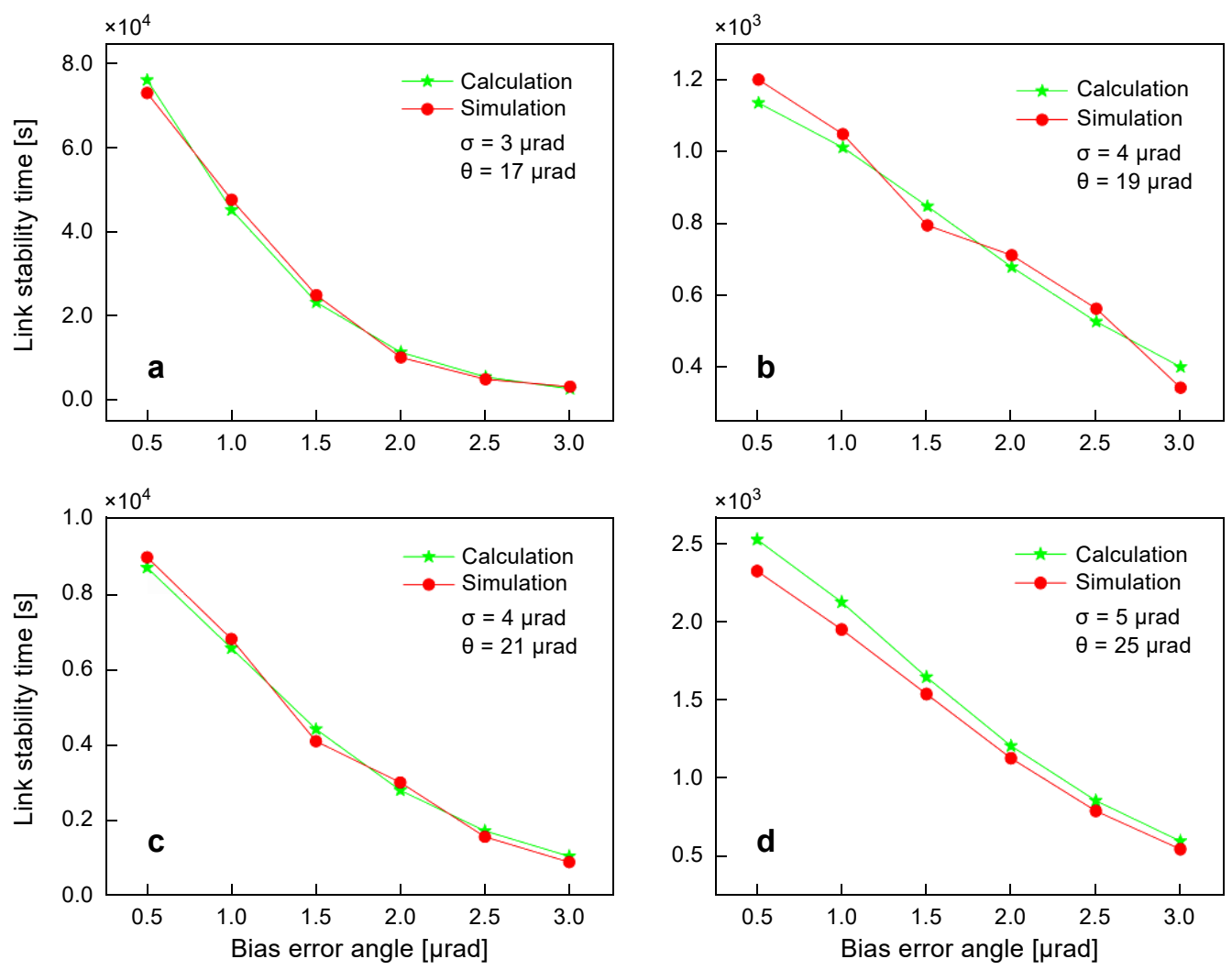

Fig. 7. Influence verification of link parameter on link lifetime; $\sigma=3 \mu \mathrm{rad}, \theta=17 \mu \mathrm{rad}(\mathbf{a}), \sigma=4 \mu \mathrm{rad}$, $\theta=19 \mu \mathrm{rad}(\mathbf{b}), \sigma=4 \mu \mathrm{rad}, \theta=21 \mu \mathrm{rad}(\mathbf{c})$, and $\sigma=5 \mu \mathrm{rad}, \theta=25 \mu \mathrm{rad}(\mathbf{d})$.

showed us that the existence of the bias error $\varphi$ can degrade the retention time of the communication system considerably. Under the premise of the same parameter value, the influence of the jitter $\sigma$ on link stability was greater than that of the static bias error $\varphi$. Obviously, to increase the beam width is an effective method to promote the communication time. The maximum and minimum of pre-pointing link retention time are 76119 and $343 \mathrm{~s}$, respectively, with the assumption that the two satellites see each other. The simulation results accorded well with the theoretical results, which proved that the theory in this paper is correct.

\section{Conclusion}

In conclusion, the 2 nd order point-ahead angle cannot be ignored in deep space ISLLs. And the static bias error angle can result in a bigger pointing error angle which can affect the link stability inevitably. It is observed that the 1st order and 2nd order point -ahead angle have reverse change regulation with the intersatellite distance. The 2 nd order point-ahead angle was a source of static bias error angle which had a weaker influence 
on communication stability than satellite jitter. Therefore, in order to increase the communication time, a vibration filter may be useful. Another solution is to promote the accuracy of the point-ahead angle and degrade the pointing error angle. Moreover, our theory has been verified in the Simulink-simulation environment. The simulation results accorded well with the numerical results. The work will improve the intersatellite laser links' design.

Acknowledgments - We would like to express my gratitude to all those who helped us during the writing of this work. This work is supported by the foundation project of Chinese Academy of Sciences (Y655811213).

\section{References}

[1] Ferdinandov E., Pachedjieva B., Bonev B., Saparev S., Joint influence of heterogeneous stochastic factors on bit-error rate of ground-to-ground free-space laser communication systems, Optics Communications 270(2), 2007, pp. 121-127, DOI: 10.1016/j.optcom.2006.09.006.

[2] Yang C., Jiang W., Rao C., Bit-error rate for free-space optical communication with tip-tilt compensation, Waves in Random and Complex Media 16(3), 2006, pp. 281-292, DOI: 10.1080/ 17455030600791678.

[3] Bailly M., Perez E., Pointing, acquisition, and tracking system of the European SILEX program: a major technological step for intersatellite optical communication, Proceedings of SPIE 1417, 1991, pp. 142-157, DOI: $10.1117 / 12.43749$.

[4] Tolker-Nielsen T., Oppenhauser G., In-orbit test result of an operational optical intersatellite link between ARTEMIS and SPOT4, SILEX, Proceedings of SPIE 4635, 2002, pp. 1-16, DOI: 10.1117 $\underline{12.464105 .}$.

[5] Yang X., Pabon L., Murry C.E., Engineering adolescence: maturation of human pluripotent stem cell-derived cardiomyocytes, Circulation Research 114(3), 2014, pp. 511-523, DOI: $10.1161 /$ CIRCRESAHA.114.300558.

[6] Fields R., Lunde C., Wong R., Wicker J., Kozlowski D., Jordan J., Hansen B., Muehlnikel G., SCHeEl W., Sterr U., Kahle R., Meyer R., NFIRE-to-TerraSAR-Xlaser communication results: satellite pointing, disturbances, and other attributes consistent with successful performance. Proceedings of SPIE 7330, 2009, article 73300Q, DOI: 10.1117/12.820393.

[7] Popescu A.F., Huber P., ReIland W., Experimental investigation of the influence of tracking errors on the performance of free-space laser links, Proceedings of SPIE 885, 1988, pp. 93-98, DOI: $10.1117 / 12.976554$.

[8] Ye X.W., SHEN F., Orbit-dynamics model of spacecraft and error analysis of ahead-point, Chinese Journal of Lasers 44(6), 2017, article 0604003.

[9] Shlomi A., KopeiKa N.S., Possible solutions to mitigate vibration effects in laser intersatellite links, Proceedings of SPIE 4489, 2002, p. 202, DOI: $10.1117 / 12.453230$.

[10] Tupper Hyde T., Porter Davis L., Vibration reduction for commercial optical intersatellite communication links, Proceedings of SPIE 3329, 1998, pp. 94-105, DOI: 10.1117/12.316883.

[11] Yu S., Wu F., Tan L., Ma J., Static position errors correction on the satellite optical communication terminal, Optical Engineering 56(2), 2017, article 026112, DOI: 10.1117/1.OE.56.2.026112.

[12] Shimizu M., Shiratama K., Ohgushi Y., Shikatani M., Arimoto Y., Aruga T., Point-ahead mechanism for ETS-VI optical ISL experiment, Proceedings of SPIE 1218, 1990, pp. 646-657, DOI: 10.1117 I $\underline{12.18224 .}$

[13] Kern R., Kugel U., Pointing, acquisition and tracking (PAT) subsystems and components for optical space communication systems, Proceedings of SPIE 1131, 1989, pp. 97-107, DOI: 10.1117 12.961537. 
[14] Greenwald D., McLaughlin C., A beacon tracker and point ahead system for optical communications, Proceedings of SPIE 1111, 1989, pp. 268-276, DOI: 10.1117/12.977987.

[15] Araki K., Inagaki K., Yasukawa K., Design of compact transceiver optical systems for optical intersatellite links, Proceedings of SPIE 1218, 1990, p. 169, DOI: $10.1117 / 12.18185$.

[16] Montenbruck O., Gill E., Lutze F.H., Satellite orbits: models, methods, and applications, Applied Mechanics Reviews 55(2), 2002, pp. B27-B28, DOI: 10.1115/1.1451162.

[17] Peters R., Sasaki M., An iterative approach to calculating the performance of two coupled optical intersatellite link tracking subsystems, Proceedings of SPIE 756, 1987, pp. 78-85, DOI: 10.1117 I 12.940027.

[18] Arnon S., Gill E., The optical communication link outage probability in satellite formation flying, Acta Astronautica 95, 2014, pp. 133-140, DOI: 10.1016/j.actaastro.2013.10.021.

[19] ARNon S., Network of sensors: acquisition probability, Journal of the Optical Society of America A, 24(9), 2007, pp. 2758-2765, DOI: 10.1364/JOSAA.24.002758.

[20] ARnOn S., Rotman S., Kopeika N.S., Beam width and transmitter power adaptive to tracking system performance for free-space optical communication, Applied Optics 36(24), 1997, pp. 6095-6101, DOI: 10.1364/AO.36.006095.

[21] Polishuk A., ARnon S., Optimization of a laser satellite communication system with an optical preamplifier, Journal of the Optical Society of America A, 21(7), 2004, pp. 1307-1315, DOI: 10.1364 / JOSAA.21.001307.

[22] Ma J., Li M., Tan L.-Y., Zhou Y.-P., Yu S.-Y., Che C., Space radiation effect on EDFA for inter -satellite optical communication, Optik 121(6), 2010, pp. 535-538, DOI: 10.1016/j.ijleo.2008.09.009.

[23] Lu W., Liu L., Sun J., Zhou Y., Wu Y., DAi E., Control analysis of acquirement and locking in inter -satellite laser communications, Optik 123(19), 2012, pp. 1750-1754, DOI: 10.1016/j.ijleo.2011. $\underline{11.097 .}$ 